\title{
Laparoscopic ICG-guided RALPPS procedure for HCC on cirrhosis with 3D reconstruction implementation: a case report
}

\author{
Francesca Pegoraro ${ }^{1}$, Roberto Montalti ${ }^{2}$, Gianluca Rompianesi ${ }^{1}$, Mariano Cesare Giglio ${ }^{1}$, Roberto Ivan \\ Troisi $^{1}$ \\ 'Division of HPB, Minimally Invasive and Robotic Surgery, Department of Clinical Medicine and Surgery, Federico II University, \\ Naples 80131, Italy. \\ ${ }^{2}$ Department of Public Health, Federico II University, Naples 80131, Italy.
}

Correspondence to: Prof. Roberto Ivan Troisi, Division of HPB, Minimally Invasive and Robotic Surgery, Department of Clinical Medicine and Surgery, Federico II University, Via Pansini 5, Naples 80131, Italy. E-mail: roberto.troisi@unina.it

\author{
How to cite this article: Pegoraro F, Montalti R, Rompianesi G, Giglio MC, Troisi RI. Laparoscopic ICG-guided RALPPS procedure \\ for HCC on cirrhosis with 3D reconstruction implementation: a case report. Hepatoma Res 2021;7:24. \\ https://dx.doi.org/10.20517/2394-5079.2020.142
}

Received: 5 Nov 2020 First Decision: 11 Jan 2021 Revised: 18 Jan 2021 Accepted: 26 Jan 2021 Available online: 8 Apr 2021

Academic Editor: Allan Tsung Copy Editor: Yue-Yue Zhang Production Editor: Yue-Yue Zhang

\begin{abstract}
We present a fully laparoscopic partial RALPPS (radiofrequency-assisted liver partition with portal vein ligation for staged hepatectomy) on a cirrhotic 71-year-old man with a bifocal hepatocellular carcinoma. The patient's liver was preoperatively studied through a CT-guided 3D-reconstruction. During stage-1, the right portal vein was ligated and injected with alcohol distally; the vascular limit between the right and left anterior sectors was defined through the systemic infusion of indocyanine green for a negative staining. Hence, laparoscopic ablations, guided by luminescence and checked with intraoperative ultrasounds, were performed. After 55 days, the future liver remnant increased from $28.6 \%$ to $46.3 \%$, allowing a laparoscopic RALPPS stage-2. Fully laparoscopic RALPPS technique shows several advantages compared to the original procedure, especially in patients with cirrhosis. The avoidance of liver transection during stage- 1 reduced blood loss and intraabdominal adhesions, and it eliminated the risk of biliary fistulae and allowed an easier liver transection during stage-2.
\end{abstract}

Keywords: Liver resection, 3D reconstruction, fluorescence, HCC, ICG, laparoscopy, LiMON test, RALPPS

\section{INTRODUCTION}

Hepatocellular carcinoma (HCC) is the fourth most common cause of cancer-related death worldwide and 
the most common hepatic primary tumor ${ }^{[1]}$. When detected at an early stage, surgery is the best curative therapy available, and the increasing understanding of liver anatomy and physiology has allowed to push the limit of resectability further over time ${ }^{[2]}$. This has been possible also thanks to the development of new technologies that assist the surgeons both preoperatively and intraoperatively.

Preoperative study implementation with CT-guided 3D reconstruction of the patient's liver has been documented in the last 20 years ${ }^{[3]}$. This approach is aimed at improving the preoperative understanding of the patient's anatomy and location of lesions, allowing the performance of an unlimited number of resection simulations to select the safest and most oncologically radical option. This technique is extremely useful in complex cases, particularly for the future liver remnant (FLR) assessment to plan radical operations while minimizing the risk of postoperative liver failure (PHLF) ${ }^{[4]}$. However, despite these advantages, $3 \mathrm{D}$ modeling applied to this setting has only been studied by few authors ${ }^{[5-7]}$.

Recently, portal vein ligation for staged hepatectomy (ALPPS) has been described as a valid technique to reduce the risk of PHLF in case of limited $\mathrm{FLR}^{\left[{ }^{[8]}\right.}$. In fact, this technique offers a rapid and consistent FLR growth, compared to portal vein embolization (PVE) ${ }^{[9-1]}$ and could also represent a salvage option in case of failed PVE. Some ALPPS variants have been developed, such as partial-ALPPS (involving a partial liver splitting), tourniquet-guided procedure (ALTPS), hybrid ALPPS and RALPPS (radiofrequency-assisted liver partition with portal vein ligation for staged hepatectomy) ${ }^{[12]}$. The RALPPS procedure, described for the first time in 2015 by Gall et al. ${ }^{[13]}$, has most of the advantages of the original ALPPS technique, while reducing some of its main drawbacks, such as the significant risks of high blood loss and biliary fistula ${ }^{[14,15]}$.

Several procedures have been developed to perform a safe liver splitting without deviating from a safe plane or causing poor FLR vascularization, such as ischemic marking of the liver surface, intraoperative ultrasound (IOUS), and indocyanine green fluorescence (ICG) $)^{[16,17]}$.

We describe a laparoscopic RALPPS procedure combined with 3D reconstruction and intraoperative ICG fluorescence.

\section{CASE REPORT}

Herein, we present the case of a 71-year-old man diagnosed with hepatitis B in the 1970s, treated with interferon. His comorbidities included reflux from hiatal hernia, chronic gastritis and benign prostatic hyperplasia, and thus, the patient took no regular medications. The preoperative blood chemistry analysis showed normal range values and did not detect HCVAb, HBsAg, HBeAb or HBsAb; HBcAb was positive and HAV IgG > $83 \mathrm{IU} / \mathrm{mL}$. Tumor markers were all within normal range (AFP $3.76 \mathrm{ng} / \mathrm{mL}, \mathrm{CEA} 1.6 \mathrm{ng} / \mathrm{mL}$, and CA $19.917 .5 \mathrm{ng} / \mathrm{mL}$ ) and the liver status was CHILD A5, MELD 7, Na-MELD 8.

During his HBV follow-up, he underwent an abdominal US that showed a hyperechoic lesion at the confluence of right and middle hepatic vein (MHV), adjacent to the inferior vena cava, of $22 \mathrm{~mm} \times 26 \mathrm{~mm}$, in Sg8-Sg4a in the context of mild to moderate steatosis. A thoraco-abdominal CT scan [Figure 1A] found two lesions with HCC characteristics, one of $45 \mathrm{~mm}$ in Sg8 and another one in Sg6 of $52 \mathrm{~mm}$ with evidence of a hyperechoic thrombosis in a peripheral portal vein branch of Sg6. A bone scintigraphy was negative for metastatic lesions.

Given the challenging position of the lesion involving the origin of the MHV and right hepatic vein (RHV), and the presence of another lesion in Sg6, complete disease eradication could have been achieved with a right hepatectomy. To better understand the lesions' position and to calculate the FLR, a virtual 

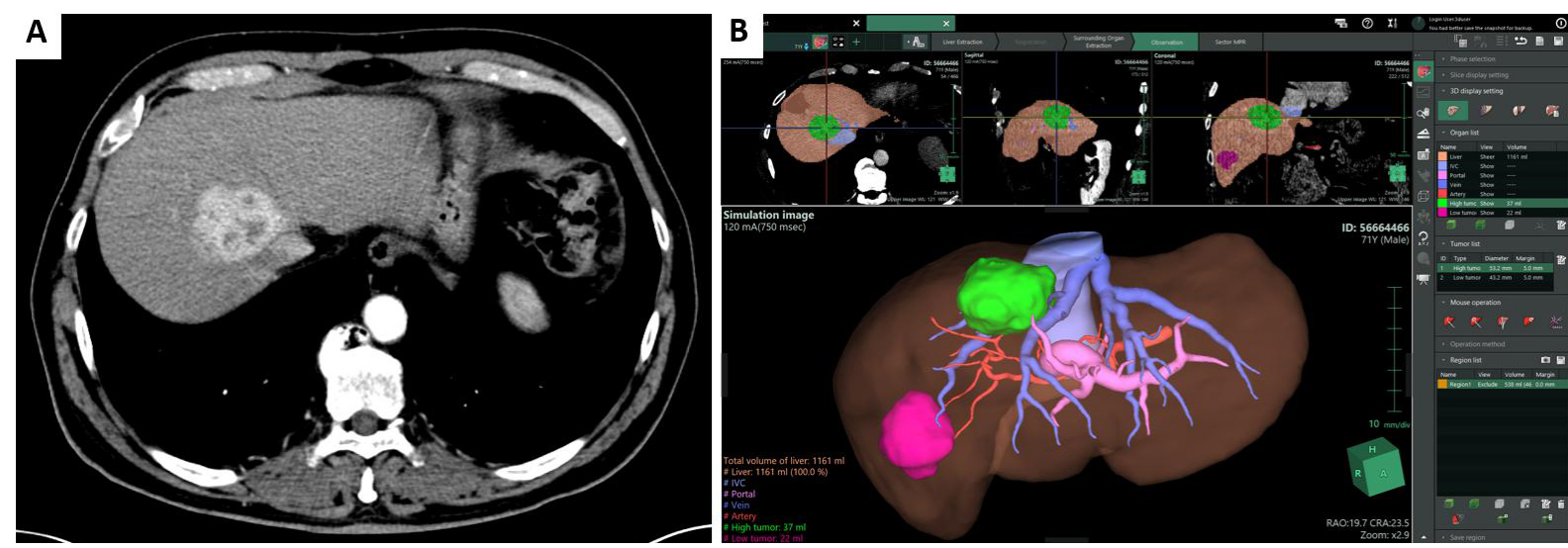

Figure 1. (A) Preoperative CT scan, showing the lesion in segment VIII next to vena cava and the displacement of right and middle hepatic veins. (B) The workstation of Synapse 3D, a software for surgical use that allows the development of virtual reconstructions based upon triphasic CT scans.

reconstruction of the liver using a software for surgical use (Synapse $3 \mathrm{D}^{\circ}$, Fujifilm;, Tokyo, Japan) was performed [Figure 1B].

We proposed two resection hypotheses, one including and one excluding the MHV from the FLR. The aim of this comparison was to evaluate whether venous drainage could be guaranteed by the left hepatic vein only. In fact, the difference between these two options in terms of FLR was rather small: the software calculated a FLR of $28.6 \%$ when conserving the MHV, vs. a $26.7 \%$ of FLR when the MHV was excluded [ Figure $2 \mathrm{~A}$ and $\mathrm{B}]$. The results of the two reconstructions are summarized in Table 1.

The multidisciplinary team evaluation concluded that the surgical ligation of the right portal branch and its distal alcoholization, with radiofrequency ablation of the liver parenchyma for right hepatectomy in two stages (RALPPS) with preservation of the MHV + cholecystectomy was the preferable option, also to reduce the surgical stress of a major resection in a cirrhotic patient.

In April 2020, the laparoscopic RALPPS stage-1 was performed. The operation started with a biopsy of the FLR, which confirmed micronodular cirrhosis. Hepatic IOUS were thoroughly performed to confirm the liver's anatomy, to identify the known lesions and their relationship with vasculo-biliary structures (the nodule in Sg8 was abutting the MHV with a clear cleavage plane and infiltrating the RHV). The right branch of the portal vein was isolated, clipped with Hem-o-Lok, transected and $18 \mathrm{~mL}$ of alcohol were injected distally [Figure 3A]. Afterwards, the right hepatic artery was temporarily clamped, and indocyanine green $(2 \mathrm{mg}$ ) was injected intravenously to mark the limit of the right liver under fluorescence guidance [Figure $3 \mathrm{~B}$ ]. The future resection plane was then ablated with nine $5-\mathrm{cm}$-deep ablations (Cool-tip ${ }^{\mathrm{m}} \mathrm{RF}$ Ablation System E Series) under IOUS guidance [Figure 3C]. The proximal right portal branch was marked with a $2 / 0$ Prolene suture, left $4 \mathrm{~cm}$ long, and a final IOUS check of the ablation plane was made. The surgical time was $275 \mathrm{~min}$, and blood loss was about $50 \mathrm{~mL}$.

The patient did not develop any postoperative complication, apart from sinus tachycardia, which regressed promptly after bisoprolol administration.

A CT scan was performed 2 days after surgery, showing complete right portal occlusion and hypodense areas of ablation, [Figure 3D]. No dilation of the biliary tract was present. The patient was discharged on 
Table 1. Volumetry of the patient's liver as reconstructed by Synapse 3D ${ }^{\circ}$ Software, before RALPP stage I and before RALPP stage II

\begin{tabular}{|c|c|c|c|}
\hline & \multicolumn{2}{|c|}{ PRE RALPP stage I } & PRE RALPP stage II \\
\hline TLV & \multicolumn{2}{|l|}{$1046 \mathrm{~mL}$} & $1220 \mathrm{~mL}$ \\
\hline NTLV & \multicolumn{2}{|l|}{$987 \mathrm{~mL}$} & $1161 \mathrm{~mL}$ \\
\hline TIV & \multicolumn{2}{|l|}{$59 \mathrm{~mL}$} & \\
\hline max diameter & \multicolumn{2}{|l|}{$51.2 \mathrm{~mm}$} & \\
\hline volume & \multicolumn{2}{|l|}{$37 \mathrm{~mL}$} & \\
\hline max diameter & \multicolumn{2}{|l|}{$43.4 \mathrm{~mm}$} & \\
\hline volume & \multicolumn{2}{|l|}{$22 \mathrm{~mL}$} & \\
\hline NTLV/TLV & \multicolumn{2}{|l|}{$94.35 \%$} & $95.16 \%$ \\
\hline TIV/TLV & \multicolumn{2}{|l|}{$5.64 \%$} & $4.83 \%$ \\
\hline \multirow[t]{2}{*}{ TIV/NTLV } & \multicolumn{2}{|l|}{$5.98 \%$} & $5.08 \%$ \\
\hline & $\begin{array}{l}\text { MHV } \\
\text { preservation }\end{array}$ & $\begin{array}{l}\text { MHV } \\
\text { excision }\end{array}$ & $\begin{array}{l}\text { Final } \\
\text { volumes }\end{array}$ \\
\hline Computed FLR & $282 \mathrm{~mL}(28.6 \%)$ & $264 \mathrm{~mL}(26.7 \%)$ & $538 \mathrm{~mL}(46.3 \%)$ \\
\hline Computed resection volume & $764 \mathrm{~mL}(71.4 \%)$ & 783 mL (73.3\%) & $682 \mathrm{~mL}(53.7 \%)$ \\
\hline Computed resection on TLV & $73.04 \%$ & $74.85 \%$ & $55.90 \%$ \\
\hline Computed resection on NTLV & $77.41 \%$ & $79.33 \%$ & $58.74 \%$ \\
\hline
\end{tabular}

TLV: Total liver volume; NTLV: non-tumoral liver volume; TIV: total lesion volume; FLR: future liver remnant; MHV: middle hepatic vein.
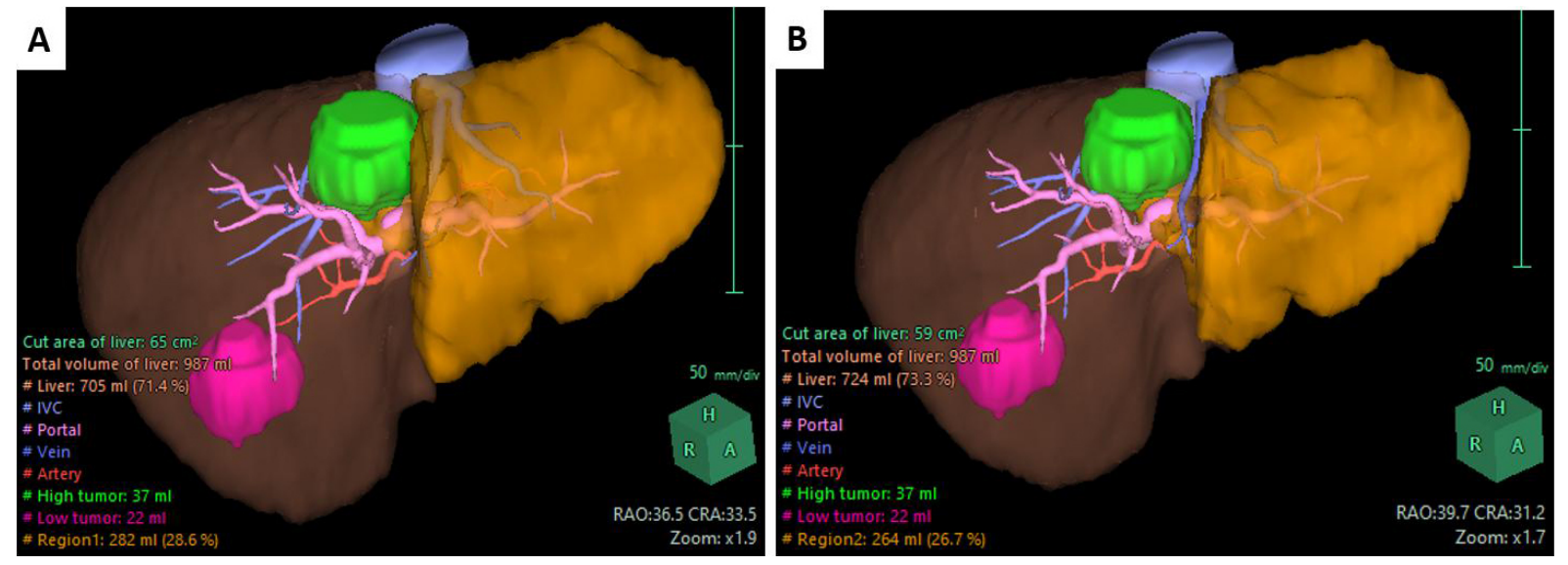

Figure 2. Preoperative computation of FLR (shaded orange) hypothesizing the conservation (A) or the exclusion (B) of the middle hepatic vein, and relative volumetries.

POD3 in good general conditions.

A new LiMON test was performed at POD23, but it showed a poor liver function (plasma disappearance rate (PDR) of $15 \% / \mathrm{min}$ and R15 of $18 \%$ ). This compelled us to wait further, despite the achievement of a good FLR hypertrophy at the control CT scan.

A new triphasic CT scan was performed 55 days after the first stage [Figure $4 \mathrm{~A}$ and B]; FLR increased to $46.3 \%$ of standard liver volume from $28.6 \%$ [Figure 4 C, Table 1]. The patient was then re-admitted and a LiMON test was performed: PDR and R15 measured $21.4 \% / \mathrm{min}$ and $4.0 \%$, respectively. We consider a PDR $>18 \% / \mathrm{min}$ and an $\mathrm{R} 15<10 \%$ as acceptable thresholds for a safe major hepatectomy, as described in the literature ${ }^{[18]}$, so the patient's fitness for a major hepatic resection was confirmed. 

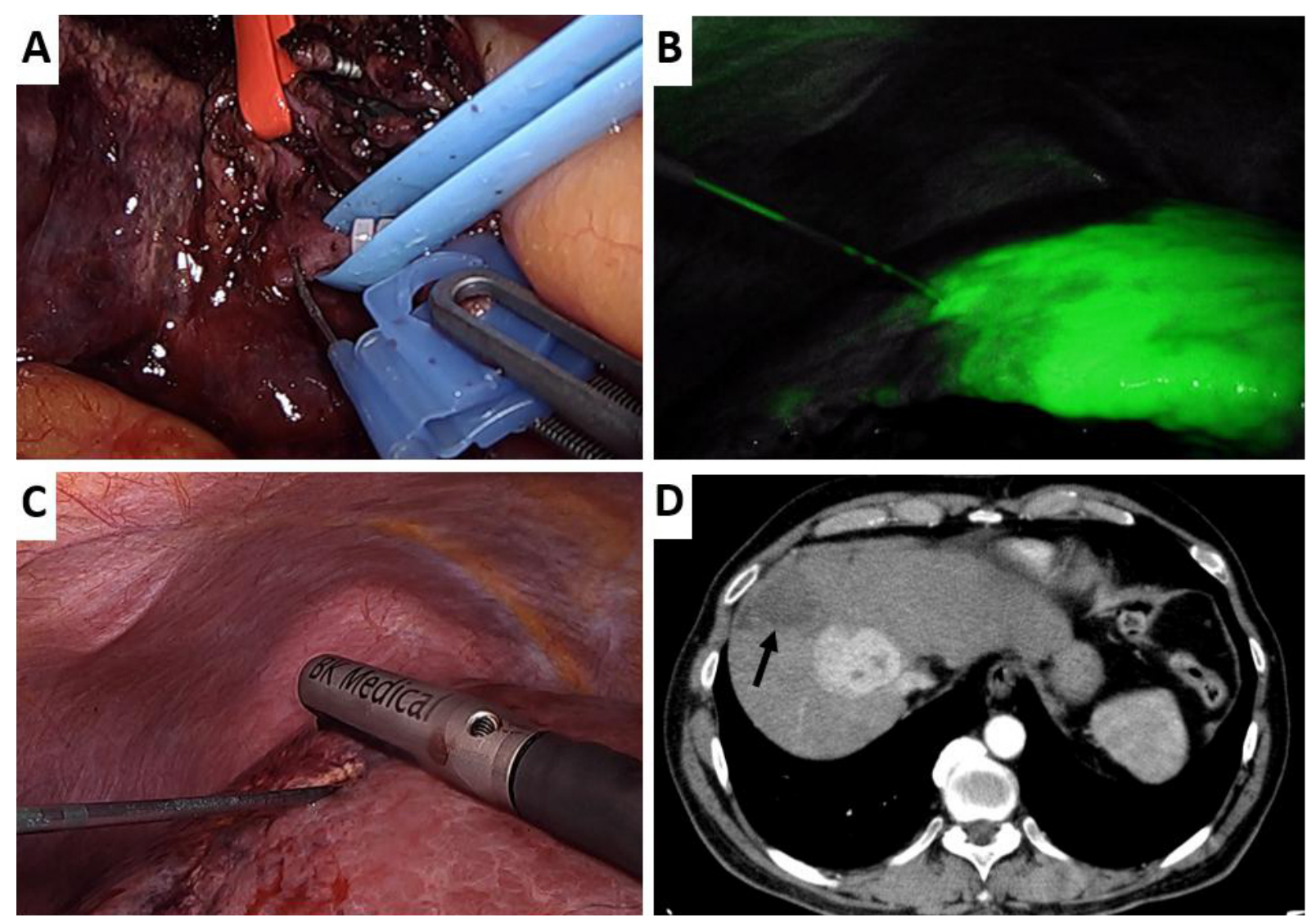

Figure 3. Intraoperative images of the RALLPS stage one. (A) Division of the right portal vein (marked with a blue vessel loop and closed proximally with a Hem-o-Lok) and $96 \%$ alcohol infusion with a butterfly needle. The right hepatic artery is surrounded by a red vessel loop and kept away from the operative field. (B) ICG-guided ablation of the future transection plane with continuous IOUS control (C). (D) Area of ablation at the control CT-scan, on POD2 (black arrow).

The next day the patient underwent a laparoscopic RALPPS stage-2 as planned. After the mobilization of the right liver up to the accessory hepatic veins and hepatocaval ligament and their section, the right hepatic vein was isolated and slinged on a vessel loop. Afterwards, the stump of the right portal branch was visualized using the Prolene 2/0 marker [Figure 5A]. The IOUS highlighted the known lesion touching the origin of the MHV. A right hepatectomy was performed with the aid of the hanging maneuver [Figure 5B] and detaching the tumor from the MHV that had been preserved, as planned to allow an adequate drainage of segment 4 [Figure $5 \mathrm{C}$ ]. The parenchymal transection was performed with CUSA Excel, and hemostasis was accomplished with titanium clips and Aquamantys [Figure 5D]. After a final hemostasis check, a CH24 Blake abdominal drain was positioned near the liver cut surface.

The total duration of the operation was 360 minutes, while blood loss was $400 \mathrm{~mL}$, and the patient did not need any intraoperative or postoperative transfusions.

The patient was admitted to the ICU and stepped down to the ward on POD1. On POD5 the patient developed hyperpyrexia $\left(38.4^{\circ} \mathrm{C}\right)$. The blood cultures did not show any growth and the CT scan showed a small left subdiaphragmatic fluid collection on the resection margin, extending caudally down to the right hepatic flexure. The patient's condition rapidly improved after the initiation of an empiric antibiotic therapy and was discharged on POD16 in good general condition. 

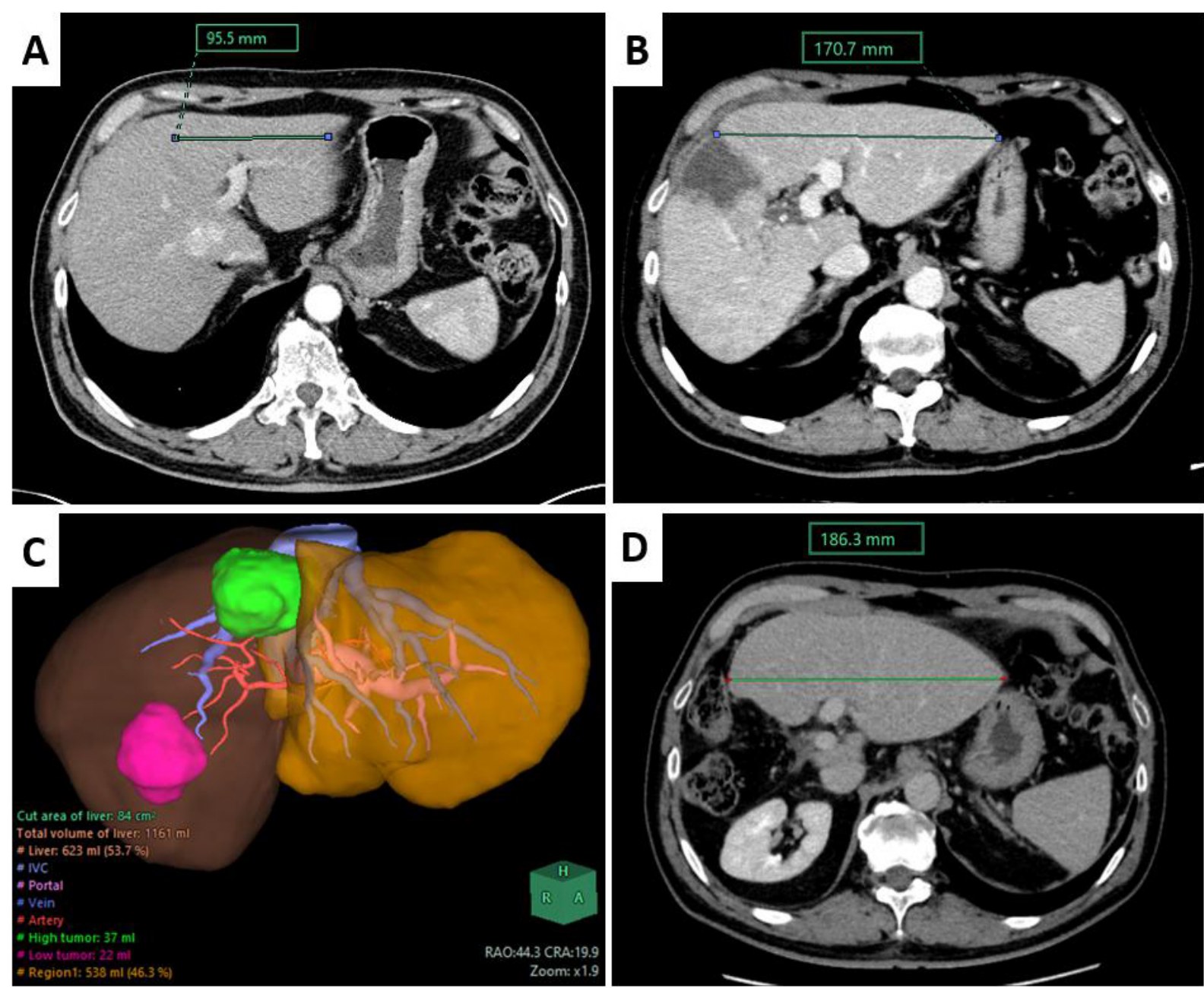

Figure 4. (A) Preoperative length of the left hepatic lobe's maximum width. (B) The same measurement in the control CT scan performed 2 days prior to RALPPS completion. (C) The 3D model based on the control CT scan, shown in B, indicates an increase in FLR from 282 to $538 \mathrm{~mL}$ (90.78\% growth rate). (D) Measurement of FLR after 6 months.

The histopathology report showed the presence of two scarcely differentiated HCC nodules of $45 \times 40 \mathrm{~mm}$ and $45 \times 35 \mathrm{~mm}$, respectively; the resection margin was $2 \mathrm{~mm}$ (Ro resection). No vascular tumoral thrombosis was present. The surrounding parenchyma was affected by minimal chronic hepatitis with ground glass modifications and polymorphic hepatocytes, to be referred to the known history of hepatitis $\mathrm{B}$ infection. Overall, the disease was staged as a pT2 Nx according to the AJCC 2017 Classification (VIII Ed.) ${ }^{[19]}$. The non-tumoral liver parenchyma was staged as an Ishak grade 2, stage $1^{[20]}$.

Six months after the completion of the RALPPS stage-2, the patient is alive and in good general condition without signs of HCC recurrence [Figure $4 \mathrm{D}$ ]. The patient approved the utilization of the present documentation for scientific purposes.

\section{DISCUSSION}

The aim of this report was to show the feasibility of a total laparoscopic RALPPS procedure in a patient with HCC and liver cirrhosis. Our endpoint was to perform a feasible and reproducible procedure, aimed at preserving as much parenchyma as possible while obtaining oncologic radicality and minimizing the risk of 

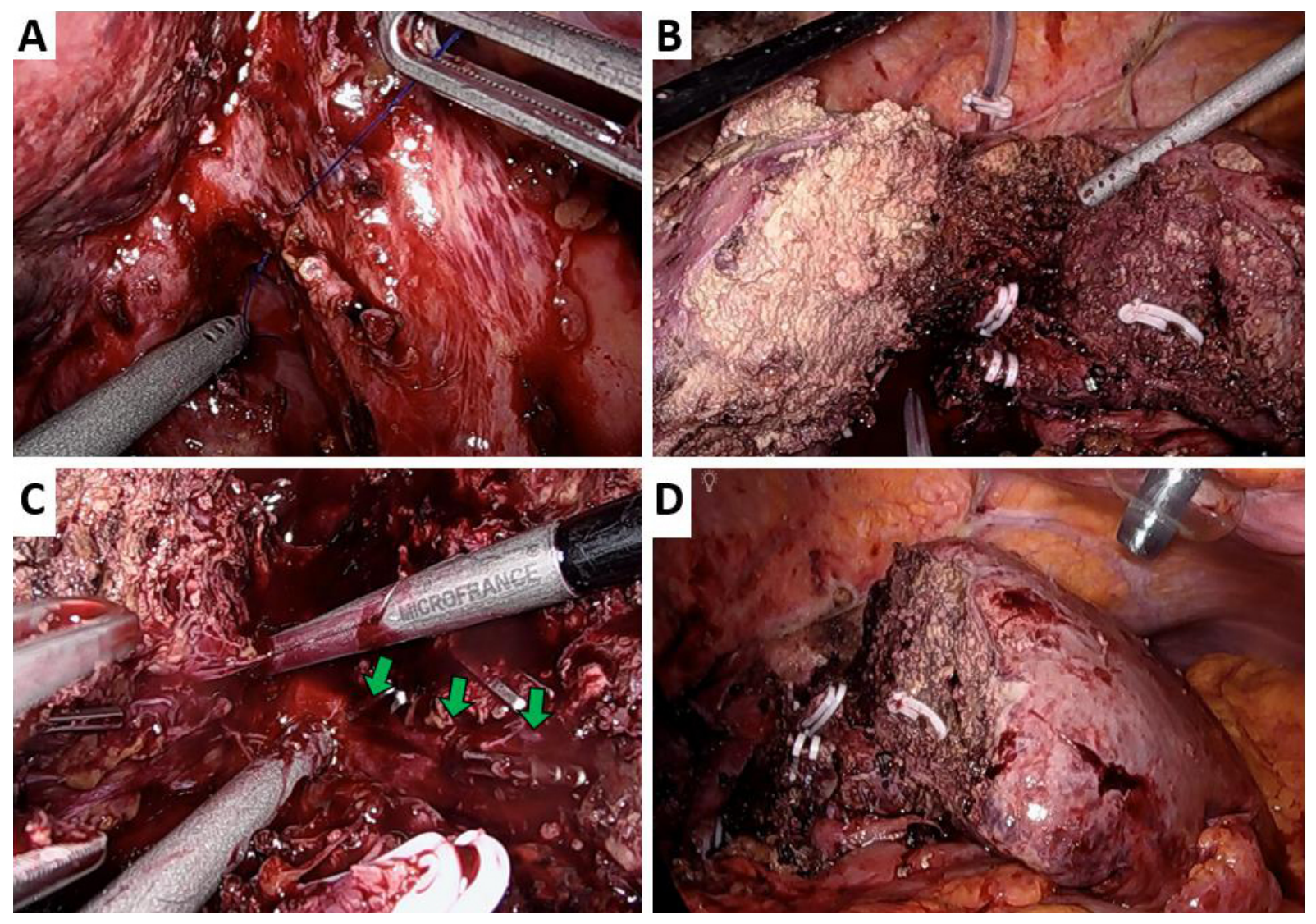

Figure 5. Intraoperative images of the RALLPS stage two. (A) Hilar dissection and isolation of the right portal vein, already sectioned during the first stage. (B) Hanging maneuver by means of a pediatric feeding tube. (C) Dissection of MHV (green arrows), left within FLR after tumor detachment. (D) Resection plane as appearing at the end of the procedure.

postoperative complications.

Despite not showing an extensive disease burden, this patient's resectability was at high risk due to the location of lesion in proximity of the hepatic veins groove. To guarantee both a complete resection and an adequate venous drainage, a right hepatectomy was deemed necessary. As previously described in several reports $^{[2,22]}$ a preservation of at least $30 \%$ of liver volume in a healthy liver is necessary to avoid PHLF, and this fraction rises to $40 \%$ when cirrhosis or cholestasis is present. A two-stage hepatectomy was excluded because compared to PVE and ALPPS, it is associated with a lower regenerative power and a higher dropout rate $^{[23,24]}$. Similarly, a portal vein embolization or ligation followed by hepatectomy was excluded due to poorer results, as recently shown in a randomized controlled trial ${ }^{[25]}$. Moreover, the proximity of one of the lesions to the MHV prompted us to complete the second stage as soon as possible to avoid neoplastic thrombosis and tumor dissemination. However, the suboptimal value of PDR and R15 at POD23 led us to wait further before carrying out the second stage.

The main controversy concerned the management of the MHV: after evaluating both possibilities, it was ultimately decided to preserve it to guarantee an adequate venous drainage of FLR.

The avoidance of the liver transection during the first stage of RALLP leads to less blood loss (and consequently less intraoperative transfusions), less postoperative complications (such as biliary fistulae, 
which often lead to intra-abdominal infections) and less adhesions ${ }^{[15]}$. This results in a double advantage during the second stage: the liver mobilization results were easier and there was limited blood loss during the resection thanks to the reduced vascularization of the resection plane guaranteed by the thermal ablation. At the same time, the ablated plane interrupts collateral blood circulation from the FLR to the liver segments including the tumor and vice-versa. This allows an increase in the FLR blood flow and the disruption of all cross-exchanges between neoplastic and healthy liver segments. These advantages add up to the benefits of a laparoscopic approach, which produces a lower inflammatory response (reducing the development of adhesions, thus facilitating the second stage), less postoperative pain and shorter length of stay $^{[26,27]}$.

Our RALPPS was accomplished by means of several percutaneous and trans-trocar passages of a single ablation needle under IOUS guidance. Despite causing a certain prolongation of the surgical time compared to using dedicated ablation devices (such as the Habib $4 \times$ bipolar resection device), in our experience, this method represents a valid alternative. In fact, the time of surgery did not exceed $300 \mathrm{~min}$, similarly to the timings reported in most series describing laparoscopic ALPPS ${ }^{[28]}$. This is also the "safe limit" above which the number of complications reported start to rise ${ }^{[29]}$.

We chose not to extend the ablation depth beyond $5 \mathrm{~cm}$ along the hypothesized resection shear, applying the same logic as a partial ALPPS; in fact, there is substantial evidence confirming that a complete resection plane is not needed to generate a sufficient hypertrophy boost ${ }^{[12,30]}$. Similarly, a partial ablation is as effective as a whole-thickness ablation plane ${ }^{[15]}$ and could be more practical and easier. This is because the wellknown heat-sink effect caused by large vessels (i.e., vena cava) could undermine and/or spread unevenly the ablation region. Secondly, deepening the ablation plane to the hilum enhances the risk of injuring important bile ducts that are very sensitive to radiofrequencies.

To further assess the exact limit between left and right hemiliver, ICG fluorescence guidance was used intraoperatively. This synthetic substance is almost exclusively metabolized by the liver and excreted by hepatocytes by means of MDR3 channels, which transport bilirubin ${ }^{[31]}$. For this reason, ICG is extremely well-tolerated and adverse reaction are anecdotal ${ }^{[32]}$. Its spectrum confers the property of fluorescence when illuminated with a 1000-1700 $\mathrm{nm}$ wavelength light, giving off a bright green light ${ }^{[33]}$. This technique is employed with several purposes in hepatobiliary surgery, such as intraoperative HCC detection after preoperative i.v. injection, intraoperative cholangiography, and liver vascularization marker. After right portal vein ligation, a systemic intravenous injection of a 2-mg bolus of ICG was performed. The substance rapidly spreads in all tissues, which temporarily appear green; after a few minutes, the background fluorescence vanishes, leaving a strong luminescence in correspondence of the liver parenchyma. With the right portal vein clamped, only the FLR appeared fluorescent, highlighting the vascular borders of left and right hemiliver: an electrocautery is then used to mark the limit of the transection/ablation plane. We kept the ablation passages slightly on the right of the identified plane, as not to affect the FLR (due to the anticipated expansion of the ablated zone on both sides of the needle).

Following this principle, ICG can be used both for positive staining (when the hemiliver, sector or segment to be resected is infused and enhanced) and negative staining (when the ICG is injected in the portal system while the pedicle of the portion to be resected is clamped). The vascular limit between the right and left anterior sectors is variable and poorly defined macroscopically: this technique allows the preservation of well-vascularized parenchyma and prevents the formation of ischemic areas. 
During the first stage of a laparoscopic RALPPS a Pringle maneuver is not necessary, as no transection and liver mobilization are performed. Some reports link the use of hilar clamping to an inferior FLR's hypertrophy, due to ischemia-reperfusion damage caused by the intracellular expression of cytokines and $\mathrm{TNF}^{[34,35]}$.

Since no transection plane was created, the apposition of foreign material between the two shears was not needed. The combination of these foresights produced only mild hilar fibrin formation and weak adhesions in correspondence of the ablation passages, allowing a safe and effective laparoscopic stage-2 completion.

The use of alcohol to achieve portal vein embolization has been previously described as a part of multimodal treatment before liver transplantation during percutaneous ethanol injection ${ }^{[36]}$ and during $\mathrm{PVE}^{[37]}$. This treatment is associated with significant periportal fibrosis and necrosis, leading to immediate blood coagulation and favoring liver hypertrophy ${ }^{[38]}$. The most common complications include pain, transient fever, decrease of platelet count, emesis, bile leak, intra/abdominal collections, liver failure, and anecdotal alcoholism and general thrombosis ${ }^{[37]}$. Our patient showed none of these complications and, on the contrary, benefited from the known hypertrophic potential of pure alcohol injection, which moreover enhanced the effect of right portal vein closure, and contributed to ablating any collateral circulation between FLR and treated liver. To our knowledge, this is the first report that describes a portal vein alcoholization during a RALPPS procedure.

After RALPPS completion, the most common complications are pleural effusion (which could cause pulmonary infection), impaired liver function, kidney failure, and ileus and abdominal collection ${ }^{[15,39]}$. The case reported herein presented a mild pleural effusion only, which was apparently not well tolerated by the patient and resulted in labored thoracic dynamics and low blood oxygen levels. The fever outbreak observed on POD5 was probably due to the development of a small subdiaphragmatic fluid collection on the resection margin. The absence of sepsis was confirmed by the blood cultures, and no further fever outbreaks were recorded after the initiation of an empiric antibiotic therapy.

At our institution we routinely use a 3D reconstruction software for surgical use (Synapse 3D'), which allows use to create a virtual model of a patient's liver and surrounding organs based on triphasic CT scan images. The purpose is to evaluate complex cases and postulate different virtual resection hypotheses taking into consideration anatomic variants, challenging lesion positions and assess possible advantages and drawbacks whenever multiple approaches are possible. In our experience, this tool application becomes even more valuable when a staged procedure is planned. The software allows us to accurately evaluate the disease burden and calculates the precise volume of each structure examined. When an optimal triphasic CT scan of 1- to 2-mm slice thickness is available, it is possible to compute each vessel's area of perfusion or drainage thanks to an automatic tool extraction. Moreover, it allows the performance of multiple virtual hepatectomies thanks to the manual resection tool, computing volumetries of each defined area accordingly. In our case, the virtual hepatectomy allowed us to better plan the right hepatectomy, considering the FLR and the relationship between the lesion and the MHV.

ALPPS and similar techniques should be applied carefully, and selection criteria must be evaluated thoroughly to avoid high morbidity and mortality as evidenced in the first ALPPS series ${ }^{[8]}$. As shown in the present report, the observance of these premises results in good outcomes and widens the limits of resectability. However, there is currently no literature available on the comparison between open and laparoscopic RALPPS. 
In conclusion, the case presented herein shows how the application of the right expertise, latest technologies and techniques allows the safe management of patients with borderline resectable disease.

This report shows that laparoscopic RALPPS for the treatment of HCC on cirrhosis is a safe and feasible procedure. ICG injection may provide additional information not only on the functional reserves but also on guiding the transection phases during the minimally invasive approaches.

In our opinion, this procedure is a valid alternative to ALPPS and to open RALPPS, especially in cirrhotic patients with expected high morbidity.

\section{DECLARATIONS}

\section{Authors' contributions}

Conceived and designed the manuscript: Troisi RI

Wrote the manuscript: Pegoraro F, Montalti R

Critically revised the manuscript: Troisi RI, Giglio MC, Rompianesi G

\section{Availability of data and materials}

Data supporting our Report are deposited in our database and are available at request via contat with our corresponding author.

\section{Financial support and sponsorship}

None.

\section{Conflict of interest}

The authors declare that they have no conflict of interests.

\section{Ethical approval and consent to participate}

At our Institution, the day before surgery all patients sign an informed consent which comprehends data collection in anonymized form (video recording included), so no external ethical approval was necessary in this case.

\section{Consent for publication}

Written informed consent was obtained from the patient before the first and the second surgery.

\section{Copyright}

(C) The Author(s) 2021.

\section{REFERENCES}

1. Burden of Disease Cancer C, Fitzmaurice C. Global, regional, and national cancer incidence, mortality, years of life lost, years lived with disability, and disability-adjusted life-years for 32 cancer groups, 1990 to 2015: a systematic analysis for the global burden of disease study. JAMA Oncol 2017;3:524-48. DOI PubMed PMC

2. EAftSot. EASL Clinical Practice Guidelines: Management of hepatocellular carcinoma. J Hepatol 2018;69:182-236. DOI PubMed

3. Hogemann D, Stamm G, Shin H, et al. Individual planning of liver surgery interventions with a virtual model of the liver and its associated structures. Der Radiologe 2000;40:267-73. DOI PubMed

4. Zhang G, Zhou XJ, Zhu CZ, Dong Q, Su L. Usefulness of three-dimensional(3D) simulation software in hepatectomy for pediatric hepatoblastoma. Surg Oncol 2016;25:236-43. DOI PubMed

5. Ho MC, Hasegawa K, Chen XP, et al. Surgery for intermediate and advanced hepatocellular carcinoma: a consensus report from the 5th Asia-Pacific Primary Liver Cancer Expert Meeting (APPLE 2014). Liver Cancer 2016;5:245-56. DOI PubMed PMC

6. Li PP, Wang ZH, Huang G, et al. Application of liver three-dimensional visualization technologies in the treatment planning of hepatic malignant tumor. Zhonghua Wai Ke Za Zhi 2017;55:916-22. DOI PubMed

7. Oldhafer KJ, Donati M, Maghsoudi T, Ojdanic D, Stavrou GA. Integration of 3D volumetry, portal vein transection and in situ split procedure: a new surgical strategy for inoperable liver metastasis. J Gastrointest Surg 2012;16:415-6. DOI PubMed 
8. Schnitzbauer AA, Lang SA, Goessmann H, et al. Right portal vein ligation combined with in situ splitting induces rapid left lateral liver lobe hypertrophy enabling 2-staged extended right hepatic resection in small-for-size settings. Ann Surg 2012;255:405-14. DOI PubMed

9. Azoulay D, Castaing D, Krissat J, et al. Percutaneous portal vein embolization increases the feasibility and safety of major liver resection for hepatocellular carcinoma in injured liver. Ann Surg 2000;232:665-72. DOI PubMed PMC

10. Imamura H, Shimada R, Kubota M, et al. Preoperative portal vein embolization: an audit of 84 patients. Hepatology 1999;29:1099105. DOI PubMed

11. Tanaka H, Hirohashi K, Kubo S, Shuto T, Higaki I, Kinoshita H. Preoperative portal vein embolization improves prognosis after right hepatectomy for hepatocellular carcinoma in patients with impaired hepatic function. Br J Surg 2000;87:879-82. DOI PubMed

12. Edmondson MJ, Sodergren MH, Pucher PH, et al. Variations and adaptations of associated liver partition and portal vein ligation for staged hepatectomy (ALPPS): many routes to the summit. Surgery 2016;159:1058-72. DOI PubMed

13. Gall TM, Sodergren MH, Frampton AE, et al. Radio-frequency-assisted Liver Partition with Portal vein ligation (RALPP) for liver regeneration. Ann Surg 2015;261:e45-6. DOI PubMed

14. Sodergren MH, Lurje G, Edmondson M, Psica A, Malago M, Jiao LR. Bi-institutional case-matched comparison of short-term clinical outcomes of radiofrequency-assisted liver partition and portal vein ligation (RALPP) and associating liver partition and portal vein ligation for staged hepatectomy (ALPPS). HPB 2016;18:e703-4. DOI

15. Wang Q, Yan J, Feng X, et al. Safety and efficacy of radiofrequency-assisted ALPPS (RALPPS) in patients with cirrhosis-related hepatocellular carcinoma. Int J Hyperthermia 2017;33:846-52. DOI PubMed

16. Torzilli G, Procopio F. State of the Art of Intraoperative Ultrasound in Liver Surgery: Current Use for Resection-guidance. Chirurgia (Bucur) 2017;112:320-5. DOI PubMed

17. Zhang P, Luo H, Zhu W, et al. Real-time navigation for laparoscopic hepatectomy using image fusion of preoperative 3D surgical plan and intraoperative indocyanine green fluorescence imaging. Surg Endosc 2020;34:3449-59. DOI PubMed

18. Gasperi A, Mazza E, Prosperi M. Indocyanine green kinetics to assess liver function: Ready for a clinical dynamic assessment in major liver surgery? World J Hepatol 2016;8:355-67. DOI PubMed PMC

19. Zhang G, Li R, Zhao X, Meng S, Ye J, Zhao L. Validation of the American Joint Committee on Cancer eighth edition staging system in patients undergoing hepatectomy for hepatocellular carcinoma: a US population-based study. J Surg Res 2018;222:55-68. DOI PubMed

20. Ishak K, Baptista A, Bianchi L, et al. Histological grading and staging of chronic hepatitis. J Hepatol 1995;22:696-9. DOI PubMed

21. Rahbari NN, Garden OJ, Padbury R, et al. Posthepatectomy liver failure: a definition and grading by the International Study Group of Liver Surgery (ISGLS). Surgery 2011;149:713-24. DOI PubMed

22. van den Broek MA, Olde Damink SW, Dejong CH, et al. Liver failure after partial hepatic resection: definition, pathophysiology, risk factors and treatment. Liver Int 2008;28:767-80. DOI PubMed

23. Chan KS, Low JK, Shelat VG. Associated liver partition and portal vein ligation for staged hepatectomy: a review. Transl Gastroenterol Hepatol 2020;5:37. DOI PubMed PMC

24. Zhou Z, Xu M, Lin N, et al. Associating liver partition and portal vein ligation for staged hepatectomy versus conventional two-stage hepatectomy: a systematic review and meta-analysis. World J Surg Oncol 2017;15:227. DOI PubMed PMC

25. Sandstrom P, Rosok BI, Sparrelid E, et al. ALPPS improves resectability compared with conventional two-stage hepatectomy in patients with advanced colorectal liver metastasis: results from a scandinavian multicenter randomized controlled trial (LIGRO Trial). Ann Surg 2018;267:833-40. DOI PubMed PMC

26. Jiao LR, Hakim DN, Gall TM, et al. A totally laparoscopic associating liver partition and portal vein ligation for staged hepatectomy assisted with radiofrequency (radiofrequency assisted liver partition with portal vein ligation) for staged liver resection. Hepatobiliary Surg Nutr 2016;5:382-7. DOI PubMed PMC

27. Rong Z, Lu Q, Yan J. Totally laparoscopic radiofrequency-assisted liver partition with portal vein ligation for hepatocellular carcinoma in cirrhotic liver. Medicine (Baltimore) 2017;96:e9432. DOI PubMed PMC

28. Michal K, Sau M, Tamara GMH, Long JR. A better route to ALPPS: minimally invasive vs open ALPPS. Surg Endosc 2020;34:237989. DOI PubMed PMC

29. Kawka M, Gall TMH, Jiao LR. Minimum invasive associating liver partition and portal vein ligation for staged hepatectomy ScienceDirect. Laparoscopic, Endoscopic and Robotic Surgery 2020;3:1-5.

30. Wu X, Rao J, Zhou X, Deng R, Ma Y. Partial ALPPS versus complete ALPPS for staged hepatectomy. BMC Gastroenterol 2019;19:170. DOI PubMed PMC

31. Cusin F, Fernandes Azevedo L, Bonnaventure P, Desmeules J, Daali Y, Pastor CM. Hepatocyte concentrations of indocyanine green reflect transfer rates across membrane transporters. Basic Clin Pharmacol Toxicol 2017;120:171-8. DOI PubMed

32. Zhai Q, Wang Y, Tian A. Severe hemodynamic instability after indocyanine green injection during off-pump coronary artery bypass grafting: a case report. Medicine (Baltimore) 2017;96:e8766. DOI PubMed PMC

33. Starosolski Z, Bhavane R, Ghaghada KB, Vasudevan SA, Kaay A, Annapragada A. Indocyanine green fluorescence in second nearinfrared (NIR-II) window. PLoS One 2017;12:e0187563. DOI PubMed PMC

34. Schadde E, Ardiles V, Robles-Campos R, Malago M, Machado M, Hernandez-Alejandro R, et al. Early survival and safety of ALPPS: first report of the International ALPPS Registry. Ann Surg 2014;260:829-36; discussion 36. DOI PubMed

35. Selzner M, Camargo CA, Clavien PA. Ischemia impairs liver regeneration after major tissue loss in rodents: protective effects of interleukin-6. Hepatology 1999;30:469-75. DOI PubMed

36. Troisi R, Defreyne L, Hesse UJ, Praet M, Decruyenaere J, De Hemptinne B. Multimodal treatment for hepatocellular carcinoma on 
cirrhosis: the role of chemoembolization and alcoholization before liver transplantation. Clin Transplant 1998;12:313-9. PubMed

37. Shimamura T, Nakajima Y, Une Y, et al. Efficacy and safety of preoperative percutaneous transhepatic portal embolization with absolute ethanol: a clinical study. Surgery 1997;121:135-41. DOI PubMed

38. Luz JHM, Gomes FV, Coimbra E, Costa NV, Bilhim T. Preoperative portal vein embolization in hepatic surgery: a review about the embolic materials and their effects on liver regeneration and outcome. Radiol Res Pract 2020;2020:9295852. DOI PubMed PMC

39. Jiao LR, Fajardo Puerta AB, Gall TMH, et al. Rapid induction of liver regeneration for major hepatectomy (REBIRTH): a randomized controlled trial of portal vein embolisation versus ALPPS assisted with radiofrequency. Cancers (Basel) 2019;11:302. DOI PubMed PMC 